\title{
Cognitive fusion: Maladaptive emotion regulation endangering body image appreciation and related eating behaviours
}

\author{
Joana Marta-Simões, M.S. \\ Cláudia Ferreira, M.S., Ph.D \\ Ana Laura Mendes, M.S.
}

Cognitive and Behavioural Center for Research and Intervention, University of Coimbra

\begin{abstract}
Body image-related cognitive fusion is described as the perception that undesired and distressing internal events related to body image are reliable representations of reality. This maladaptive emotion regulation process is known for its impact on human suffering, namely eating psychopathology. On the contrary, body appreciation is a positive body image construct, defined as an attitude of acceptance and affection toward one's body image characteristics, despite some level of dissatisfaction. Although body appreciation associates negatively with several pathogenic processes, its relationship with body image-related cognitive fusion is still unexplored. The present study intended to analyse the power of body image related cognitive fusion regarding its impact on body appreciation, and also on the engagement in disordered eating. The study's sample comprised 308 women, aged between 18 and 35. A path model tested the mediator role of body image related cognitive fusion in the relationship between body image dissatisfaction and body appreciation, and eating disorders symptomatology, controlled for the effect of body mass index.
\end{abstract}


The tested model accounted for $67 \%$ of the variance of disordered eating. Body image related cognitive fusion appeared as a significant mediator of the association between body dissatisfaction and body appreciation. Body appreciation presented a significant direct effect on eating disorders symptomatology. These findings highlight the disturbing effect of cognitive fusion related to body image on the construction of a positive body image, presenting an impact on the engagement in disordered eating. In this line, cognitive fusion may be a pertinent target in clinical practice and also within eating disorders prevention. 